\title{
MISCELANEA
}

PRIMERAS JORNADAS CIENTÍFICAS DE ESTUDIANTES DE ENFERMERÍA. FACULTAD CIENCIAS DE LA SALUD. UNIVERSITAT JAUME I. CASTELLÓN DE LA PLANA.

\section{APROXIMACIÓN ENFERMERA A LOS CUIDADOS NO PROFESIONALES A TRAVÉS DE LAS VIVENCIAS DE UNA CUIDADORA}

Autores: Arce Delgado S; Morgoci R; Escrig Sos L.

Correo electrónico: s.arce1@hotmail.com

Institución: Escuela de Enfermería "Ntra Sra. Sgdo. Corazón"-Castellón.

Tipo de comunicación: oral

Introducción: El envejecimiento progresivo de la población española, y la creciente disminución de los recursos institucionales para el cuidado de las personas dependientes, implica que cada día sea mayor la necesidad de disponer de cuidadoras no profesionales. En la mayoría de los casos la calidad de vida de las personas dependientes depende de los cuidados no 
profesionales prestados por mujeres y familiares en general de la persona dependiente. Cuidados y cuidadores son a menudo invisibilizados y/o poco reconocidos desde el punto de vista social, institucional y legislativo. Es función de enfermería conocer esta problemática, identificar a las cuidadoras no profesionales y las necesidades derivadas de su rol, y dispensarles la ayuda, conocimiento y apoyo necesario para realizar esta tarea en las mejores condiciones posibles.

Objetivos: Definir los conceptos relacionados con "el cuidar" dentro del sector popular de la salud para poder comprender las vivencias y la problemática de las personas que ejercen de cuidadoras dentro de este sector. Analizar e interpretar el discurso de una cuidadora informal relacionándolo con las categorías diagnósticas propuestas por la NANDA para poder descubrir los problemas relacionados con el cuidado donde sería posible la intervención enfermera al cuidador.

Material y métodos: Revisión bibliográfica sistemática con inclusión de textos legislativos relevantes para este trabajo. Entrevista semiestructurada a una cuidadora no profesional. Análisis del discurso. Codificación abierta e inductiva de la información con establecimiento de dominios y categorías de análisis, tomando como modelo la "Teoría Fundamentada" de Glaser y Strauss. Resultados: dominios y categorías diagnósticas NANDA en base al discurso de una cuidadora.

Conclusiones: Dada la importancia los cuidados no profesionales es fundamental que los programas de atención sanitaria de nuestra cultura y nuestra sociedad, creen las bases para garantizar el bienestar biopsicosocial de la cuidadora no profesional.

Por otra parte, las cuidadoras no profesionales sufren numerosos problemas de salud en los que el profesional de enfermería puede actuar, especialmente detectando y previniendo los factores de riesgo para el cuidador. 